\title{
Fixation of subtrochanteric fractures
}

\section{Does a technical optimization of the dynamic hip screw application improve the results?}

\author{
Elsayed Ibraheem Elsayed Massoud
}

Received: 23 June 2008 / Accepted: 4 May 2009/Published online: 6 June 2009

(C) Springer-Verlag 2009

\begin{abstract}
The subtrochanteric region has certain anatomical and biomechanical features that can make fractures in this region difficult for the treating surgeon. The preferred type of device is a matter of debate. Increased understanding of mechanical characteristics of the dynamic hip screw (DHS) has reduced the incidence of complications. Our hypothesis is based on the technical optimization of the DHS application. We prospectively studied 37 patients with subtrochanteric fractures with a mean age of 42.9 years. We utilized a two-stage protocol: initially, conversion of the comminuted fractures into two part fractures; then application of the implant with a technique that allowed dynamization of the DHS. Clinical and radiographic data were used to assess the outcome at 12 months. Fracture healing was obtained for all cases in a mean time of 11.64 weeks. One patient had $1.5 \mathrm{~cm}$ shortening of the injured limb. No implant failure was reported. All patients resumed pre-injury activities of daily living. It was concluded that the patients who were treated with the technical optimization of the DHS application achieved a close-to-normal anatomy following surgery and maintained this state throughout the follow-up period.
\end{abstract}

Keywords Subtrochanteric fracture ·

Dynamic hip screw $\cdot$ Biomechanics

E. I. E. Massoud ( $₫)$

Sohage Teaching Hospital, Sohage, Egypt

e-mail: Elsayedmassoud@Hotmail.com

E. I. E. Massoud

Elgehad St., Tahta, Sohage, Egypt

\section{Introduction}

Fractures in the subtrochanteric region are difficult to treat because of their anatomical and biomechanical features $[1$, 2]. Restoration of femoral length and rotation and correction of femoral head and neck angulation to restore adequate abductor tension and strength are essential to regain maximal ambulatory capacity [3]. This can be achieved with operative treatment, but no single implant is universally recommended.

The dynamic compression hip screw (DHS) has been a popular method of internal fixation for subtrochanteric fractures [3]. It provides compression along the femoral neck, and if the reduced fracture is stable, load-sharing between the bone and implant can occur [1, 4]. However, if the fracture is not stable, progressive medial displacement of the femoral shaft can occur, which may result in fixation failure and nonunion. Failures increased sevenfold, if medialization of more than $1 / 3$ of the femoral diameter at the fracture site occurred $[4,5]$. The concept of the DHS with a trochanteric stabilizing plate is to prevent or reduce medial displacement [6]. However, if the trochanteric stabilizing plate impedes further compression of the fracture before the fracture has become stable, the fracture may angulate into varus with lag screw cut-out, loosening or breaking the plate as a result [4]. The Medoff sliding plate (MSP) evolved from the DHS and gives axial compression along both the femoral neck and shaft. It produced excellent results in one prospective trial on subtrochanteric fractures [4]. However, Miedel et al. [7] reported a high rate of failure when the MSP was used in fixation of subtrochanteric fractures.

This debate about modification of the DHS has encouraged us to search for the best technical way to use the DHS in all types of subtrochanteric fractures. Our 
proposal is based on a two-step technical optimization of the DHS application: first, we reconstitute the medial buttress through conversion of the comminuted fractures into two part fractures. This stabilizes the fracture and prevents progressive medialization of the femoral shaft. Second, we enhance the sliding capacity of the DHS by fixing the plate with a technique that avoids blocking dynamization. This procedure does not prevent further compression of the stabilized fracture and protects against angulation into varus or breakage of the plate.

In this study, we present the outcome of technical optimization of the DHS application in fixation of subtrochanteric fractures.

\section{Patients and methods}

Between October 2002 and February 2007, we prospectively treated 37 patients with subtrochanteric fractures who agreed to participate in the study (Table 1). We excluded those with a pathological fracture. Plain radiographs were obtained on admission, including antero-posterior (AP) pelvis. AP and lateral plain radiographs of the entire femur were also obtained to decide on a suitable plate length.

The primary assessment included categorization of the fractures according to the Seinsheimer classification [2] (Fig. 1). The patients were interviewed about their mobility and activities of daily living (ADL) during the week before the fracture as baseline data. The Katz ADL index [8] status is based on an evaluation of the functional independence or dependence of patients in bathing, dressing, going to the toilet, transferring, continence, and feeding. Index A indicates independence in all six functions. B indicates independence in all but one of the six functions. $\mathrm{C}-\mathrm{G}$ indicate
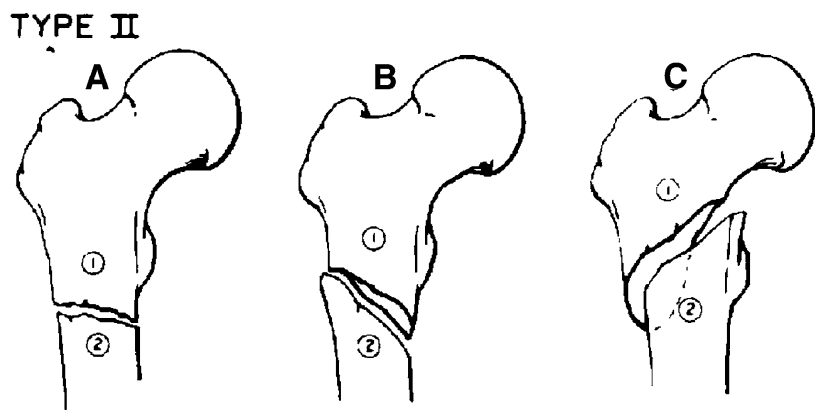

TYPE III
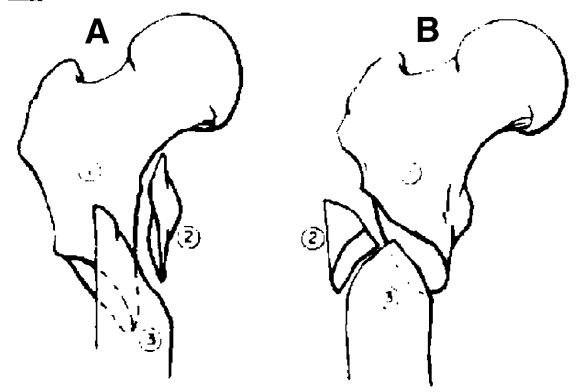

TYPE IZ

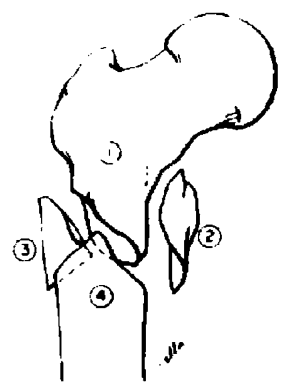

TYPE $\mathbb{Z}$

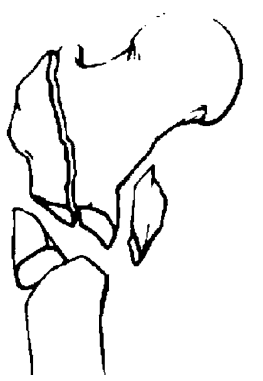

Fig. 1 The Seinsheimer's classification of subtrochanteric fractures (N.B type I: nondisplaced fractures; any fracture with less than $2 \mathrm{~mm}$ of displacement of the fracture). Reprinted from [2] with permission from the Journal of Bone and Joint Surgery, Inc

Table 1 Preoperative data for the patients with subtrochanteric fractures

\begin{tabular}{|c|c|c|c|c|c|c|c|c|c|c|c|}
\hline \multirow[t]{2}{*}{ Type } & \multicolumn{2}{|c|}{ Age (years) } & \multicolumn{2}{|l|}{ Sex } & \multicolumn{2}{|l|}{ Side } & \multicolumn{2}{|c|}{ Trauma } & \multicolumn{2}{|c|}{ Time (days) } & \multirow[t]{2}{*}{ Total } \\
\hline & Range & Average & Male & Female & Right & Left & High & Low & Range & Average & \\
\hline I & 0 & 0 & 0 & 0 & 0 & 0 & 0 & 0 & 0 & 0 & 0 \\
\hline IIA & $19-76$ & 47.5 & 1 & 1 & 1 & 1 & 1 & 1 & $2-4$ & 3 & 2 \\
\hline IIB & $21-66$ & 49.4 & 4 & 1 & 1 & 4 & 3 & 2 & $2-5$ & 4 & 5 \\
\hline IIC & 0 & 0 & 0 & 0 & 0 & 0 & 0 & 0 & 0 & 0 & 0 \\
\hline IIIA & $58-79$ & 68 & 3 & 0 & 3 & 0 & 0 & 3 & $2-5$ & 4 & 3 \\
\hline IIIB & $20-56$ & 38.27 & 9 & 2 & 7 & 4 & 8 & 3 & $1-10$ & 3.18 & 11 \\
\hline IV & $19-76$ & 34.9 & 11 & 1 & 5 & 7 & 11 & 1 & $1-8$ & 3.16 & 12 \\
\hline V & $41-75$ & 50.75 & 4 & 0 & 1 & 3 & 2 & 2 & $2-8$ & 4.25 & 4 \\
\hline Total & $19-79$ & 42.9 & 32 & 5 & 18 & 19 & 25 & 12 & $1-10$ & 3.45 & 37 \\
\hline
\end{tabular}

Type type of subtrochanteric fracture according to Seinsheimer classification, time time that elapsed before surgery 
dependence in bathing and at least one other function. Walking ability was classified into two categories: able to walk independently without aids and walking independently with one walking aid.

\section{Operative technique}

We used a radiolucent operative table, with an image intensifier; the patient was placed supine and the limb draped free. The ipsilateral skin of the iliac crest was prepared in expectation of bone grafting. Under spinal or general anaesthesia, we made a straight lateral incision from the greater trochanter, extending distally along the lateral side of the thigh, for a length determined by the length of the side plate required. The steps of fracture reduction were modified according to the fracture category. In three part or comminuted fractures (types IIIA, IIIB, IV subtrochanteric fractures and comminuted type $\mathrm{V}$ subtrochanteric-intertrochanteric fractures), we converted the comminuted fractures into two part fractures with interfragmentary screws (Fig. 2a, b). Draping the limb free facilitated knee flexion, and hip flexion and rotation, permitting dissection through relatively relaxed muscles, which helps preservation of soft tissue attachment to the bone fragments. A third fragment comprising the lesser trochanter with a small cortical extension is inaccessible. Therefore we ignore it provided that no gap is left medially (Fig. 3a, b); otherwise a cortico-cancellous graft is inserted. All fractures are transformed into two part subtrochanteric fractures, and a
DHS inserted using the standard technique with the plate fixed to the distal fragment using cortical screws. The plate holes overlying the proximal fragment are left empty (Fig. 2b, c). In an attempt to increase axial compression through the plate at the fracture site, we insert at least two of the cortical screws eccentrically in the distal plate holes. The incision is closed over a suction drain.

\section{Follow-up}

Postoperative management is tailored to the quality of the reduction. Strengthening exercises for the entire lower extremity and range-of-motion exercises are begun immediately. After an X-ray examination on the first postoperative day, regardless of the fracture category, the patients are allowed to walk with crutches, toe touching until pain disappears and good callus formation has been observed on radiographs. Then, progressive weight bearing is started; but if the reduction is not considered good, partial weight bearing is allowed when the callus bridges the gaps.

All patients receive a postoperative prophylactic antibiotic for 5 days, and are treated with low molecularweight heparin during their stay in hospital. They are discharged when mobile and primary complications have been excluded.

Follow-up reviews are undertaken at 6, 8, 10, and 12 weeks; then 4, 6, and 12 months. After the first year, patients were observed and re-evaluated twice per year. For sameness, clinical outcome was assessed with data at 12
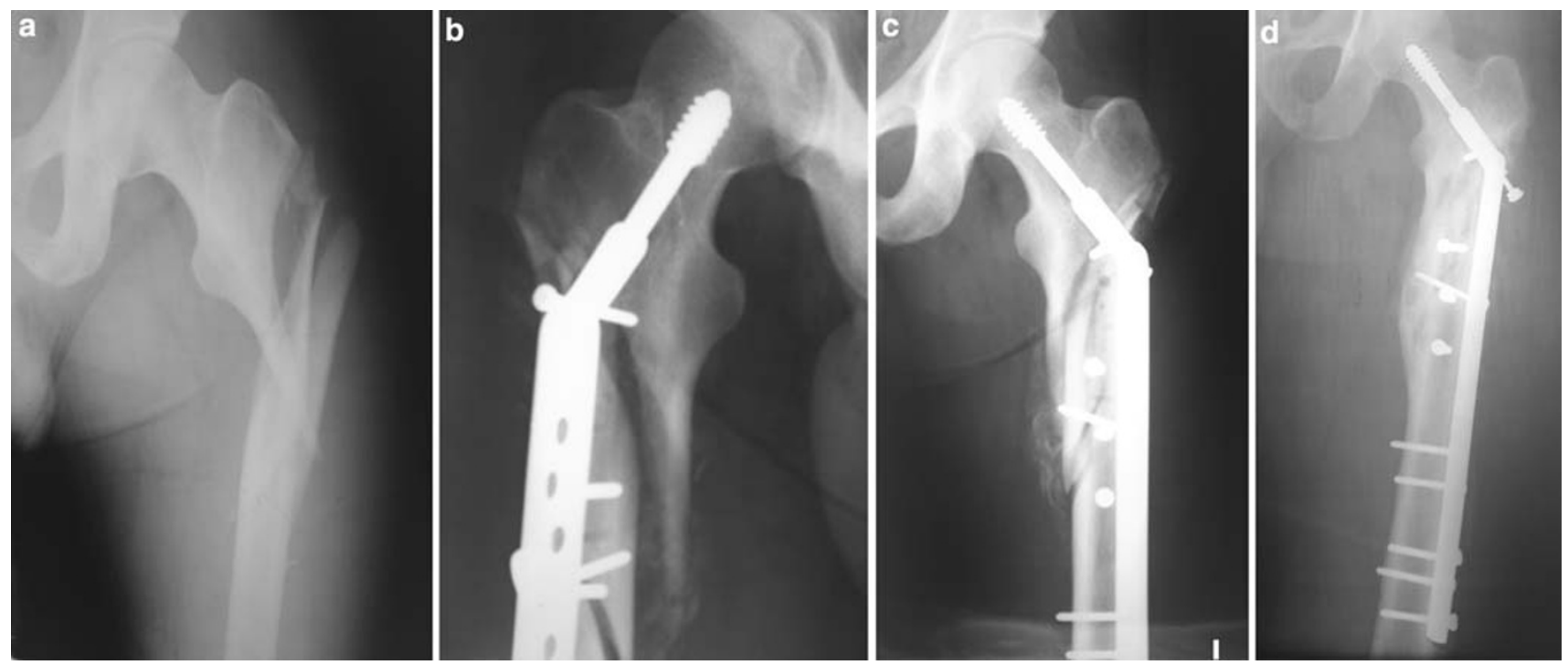

Fig. 2 a Preoperative A-P radiograph for 37-year old man. The right femur shows a type IV subtrochanteric fracture. b Immediate postoperative radiograph shows a longitudinal gap exceeding $2 \mathrm{~mm}$ between the proximal and the distal fragment. c Radiograph 2-weeks postoperatively. Because application of the DHS was closest to the hip joint reaction force and the plate was not fixed to the proximal fragment, the dynamization of the DHS is not blocked. Therefore, the gap reduced, the fracture stabilized and callus formation started medially. d Radiograph 1-year postoperatively shows healed fracture and no implant failure 

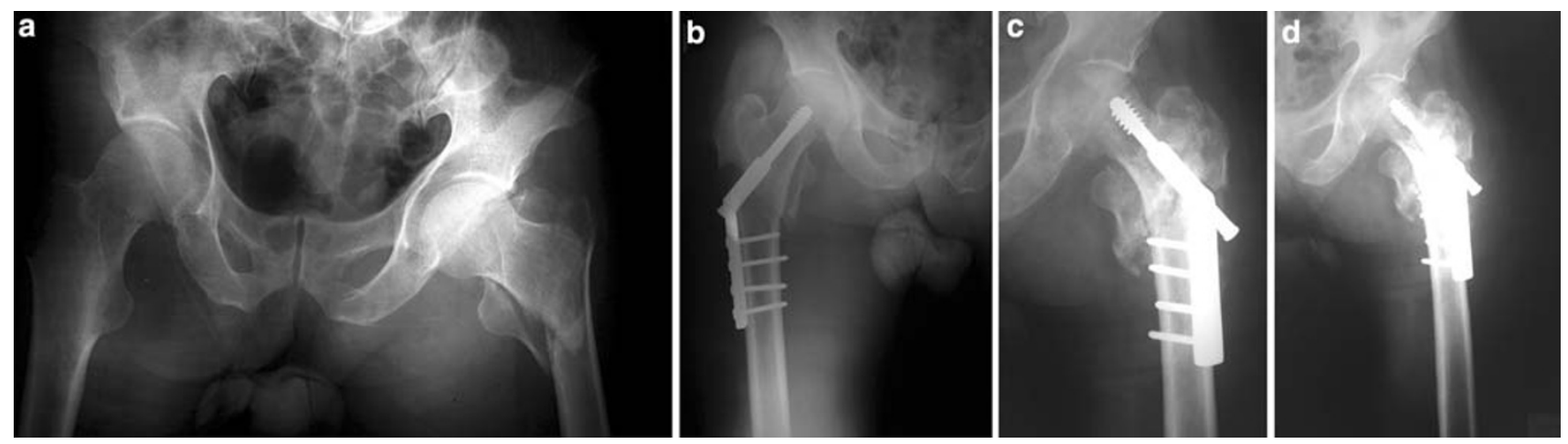

Fig. 3 a Preoperative A-P radiograph of the pelvis and both hip joints for 75-year old man. The right femur shows a type $\mathrm{V}$ subtrochanteric-intertrochanteric fracture. b Immediate postoperative radiograph: the plate is not fixed to the proximal fragment. Although the fragment that contains the lesser trochanter is not incorporated to any of the main fragments but medial bone-to-bone contact is

months. Plain AP and lateral radiographs are obtained at all visits. Hip and knee joint motion are measured with a goniometer and compared to the uninjured side. Leg length is assessed clinically on both sides by measuring the distance between the anterior superior iliac spine and the tip of the medial malleolus.

The radiological outcome includes assessment of the reduction of the fracture, fixation failure and fracture healing. Reduction is categorized as good if the femoral neck-shaft angle is $<10^{\circ}$ of varus or $<15^{\circ}$ of valgus when compared with the uninjured contralateral hip, and the displacement between the fragments does not exceed $2 \mathrm{~mm}$ in any projection [9]. Fixation failures are defined as lagscrew penetration or cut-out of the femoral head, breakage or loosening of the plate or nonunion of the fracture. Migration of the lag screw in the femoral head or varus angulation of the fracture, without lag-screw penetration or cut-out, is not regarded as fixation failure [4]. The time to union is calculated from the day of surgery to the date of achieved during reduction of the fracture. c Radiograph 10-weeks postoperatively shows the reduction is maintained, sliding of the lag screw within the barrel of the plate has occurred, and callus bridges the fracture fragment. d Radiograph after weightbearing, showing evident sliding of the lag screw and no implant failure

bone healing, which is assumed when the fracture gap is not visible on the two views of plain radiographs.

\section{Results}

The base line data for the 37 patients included in the study are listed in Table 1. According to the Katz ADL index [8] for the pre-fracture period, $33 / 37(89.2 \%)$ of the patients were classified as index A and 4/37 (10.8\%) of the patients as index B. $34 / 37$ (91.9\%) of the patients were able to walk independently without aids and $3 / 37(8.1 \%)$ of the patients with one walking aid.

In the immediate postoperative radiograph, reduction was considered good in 35/37 (94.6\%) of the patients (Table 2). In 2/37 (5.4\%) of the patients reduction was considered to be not good, because $>2 \mathrm{~mm}$ displacement between the fragments was detected in one view (cases no. 1,6$)$.

Table 2 The results of subtrochanteric fractures treated with the DHS

\begin{tabular}{|c|c|c|c|c|c|c|c|c|c|}
\hline \multirow[t]{2}{*}{ Type } & \multicolumn{2}{|c|}{ Quality of reduction } & \multicolumn{2}{|c|}{ Time to union (weeks) } & \multicolumn{2}{|c|}{ Leg length } & \multicolumn{2}{|c|}{ Follow up period (months) } & \multirow[t]{2}{*}{ Total } \\
\hline & Good & Not good & Range & Average & Equal & Shortening & Range & Average & \\
\hline I & 0 & 0 & 0 & 0 & 0 & 0 & 0 & 0 & 0 \\
\hline IIA & 2 & 0 & $8-10$ & 9 & 2 & 0 & $12-24$ & 18 & 2 \\
\hline IIB & 5 & 0 & $8-16$ & 12.2 & 5 & 0 & $12-24$ & 18 & 5 \\
\hline IIC & 0 & 0 & 0 & 0 & 0 & 0 & 0 & 0 & 0 \\
\hline IIIA & 3 & 0 & $8-12$ & 10 & 3 & 0 & $12-24$ & 18 & 3 \\
\hline IIIB & 11 & 0 & $8-16$ & 10.5 & 11 & 0 & $12-24$ & 19 & 11 \\
\hline IV & 11 & 1 & $8-20$ & 11.8 & 12 & 0 & $12-30$ & 19.3 & 12 \\
\hline V & 3 & 1 & $12-20$ & 16 & 3 & 1 & $12-24$ & 17.5 & 4 \\
\hline Total & 35 & 2 & $8-20$ & 11.64 & 36 & 1 & $12-30$ & 18.7 & 37 \\
\hline
\end{tabular}

Type type of subtrochanteric fracture according to Seinsheimer classification 
Case no. 1: The patient was 37 years old, with a type IV fracture (Fig. 2a). Because of $>2 \mathrm{~mm}$ displacement between the fragments (Fig. 2b), he was instructed to avoid postoperative weight bearing. At end of the second week, the fracture gap reduced and callus was evident medially in the radiograph (Fig. 2c); therefore, partial weight bearing was allowed. At the ninth week, the fracture had healed and full weight bearing was allowed. At the end of the followup period (Fig. 2d), no implant failure and no infections were reported.

The femoral neck-shaft angle was measured in the A-P radiograph; all patients reported no differences between both sides and this persisted to the end of the follow-up period. All fractures united within an average of 11.64 weeks (8-20 weeks).

At the final assessment, hip and knee joint motion were the same on both sides. Equal lower limb lengths were found in 36/37 (97.3\%) of the patients and leg-length discrepancy was reported in $1 / 37(2.7 \%)$ of the patients (Table 2).

The number of patients who were using one cane for walking increased from $3 / 37(8.1 \%)$ of the patients prior to injury to $4 / 37(10.81 \%)$ at final assessment. There were no differences in the ADL status between the pre-fracture and final assessment periods.

\section{Complications}

There were no cut-outs, breakage or pull-out of screws. There were no re-operations in any of the patients during the study. General complications (cardiac, pulmonary, thromboembolic or cerebrovascular) were not seen.

Superficial wound infection occurred in the second postoperative week in 2/37 (5.4\%) patients. The infection was controlled within 5 days by using daily wound dressing and antibiotics.

Shortening $1.5 \mathrm{~cm}$ of the injured limb compared to the contralateral limb was reported in one patient (case no. 6). At the time of operation, the patient was 75 years old with a type $\mathrm{V}$ subtrochanteric-intertrochanteric fracture (Fig. 3a). According to the study protocol, the inaccessible third fragment consisting of the lesser trochanter with a small cortical extension was not integrated with any of the two main bone fragments. As there were no palpable gaps and none medially visible with the image intensifier, no bone grafts were used. In one view of the immediate postoperative radiographs, $>2 \mathrm{~mm}$ displacement between the fragments was detected (Fig. 3b). Excessive impaction (collapse) at the fracture site and excessive sliding of the lag screw were seen in subsequent radiographs (Fig. 3c, d). Healing occurred at the fourth month postoperatively.

\section{Discussion}

Successful treatment of subtrochanteric fractures depends on stable osteosynthesis. However, because subtrochanteric fractures are often comminuted, stable internal fixation can be difficult to achieve [10]. Also, these fractures extend into diaphyseal bone, which has decreased vascularity and, therefore, poorer healing potential [2]. This predisposes internal fixation of these fractures to high rate of failure. Kyle reported that the compression strains are considerably greater than the tension strains [1]. These large stresses on the medial cortex in the subtrochanteric area make cortical restoration at the time of surgery mandatory to prevent cyclic loading and failure of any device used on the tension side of the femur [10].

The development of newer techniques and devices has simplified the treatment of subtrochanteric fractures. Intramedullary nailing lacks popularity compared to the DHS, and variability of the results correlates to experience in the surgical technique [7]. The most widely used fixation implant for proximal femoral fractures is probably still the DHS. To use it correctly in a patient who has a subtrochanteric fracture, it is essential to understand the mechanics of the device and the forces that it must withstand [1].

Although the side plate of the DHS has increased strength, this does not nullify the importance of medial buttress reconstitution, because even the most massive plate will undergo fatigue failure [10]. Our study protocol recommended construction of the medial buttress through conversion of a comminuted fracture into a two-part fracture. The proximal part consists of the head, neck, trochanter(s), and their distal extension to the nearest fracture line. Using interfragmentary screws, the comminuted distal fragment(s) are secured to each other and to the main diaphyseal fragment, thereby rebuilding the distal part of the subtrochanteric fracture. When there is an inaccessible lesser trochanter as a third fragment, we ignore it if there is no medial gap; otherwise a cortico-cancellous graft should be inserted $[1,10,11]$. Therefore, when the distal and proximal fragments are reduced, medial bone-to-bone contact is restored and the fracture stabilized. Otherwise, progressive medial displacement of the femoral shaft can occur, which may result in fixation failure and nonunion $[4,5]$.

Draping the limb free facilitates knee flexion and hip joint flexion and rotation [12], permits dissection through relatively relaxed muscles and helps reduction and screw fixation of the comminuted fragments. Also, preservation of soft tissue attachments to the comminuted bone fragments retains their vascularity and callus-forming properties [3]. All fractures united in an average of 11.64 weeks, 
which suggests that medial dissection did not delay bone healing.

The large proximal screw in the compression hip screw system obtains excellent purchase in the proximal fragment [11], and the ability of the screw shaft to slide in the collar of the plate allows for impaction of the fracture surfaces [10]. The sliding also permits slight medial displacement of the shaft in relationship to the proximal fragment. This tends to reduce the bending moment and the resulting forces that lead to collapse of the medial buttress and varus displacement $[1,10]$. For this sliding to occur, the plate must not be fixed with screws into the proximal fragment [10] (Figs. 2, 3). The beneficial effect of this technical suggestion has been confirmed in the current study. In one of our patients, a longitudinal gap $(>2 \mathrm{~mm})$ in the medial buttress was detected in the postoperative radiograph. The unblocked dynamization of the DHS obliterated this gap through slight medialization of the distal fragment [10] (Fig. 2a-d). Similarly, Lunsjö et al. [4] reported loosening of the plate in a well-reduced subtrochanteric fracture fixed with the dynamic hip screw with trochanteric stabilizing plate (DHS/TSP); they suggested that the trochanteric stabilizing plate impeded lag screw dynamization.

Surgeons who used the MSP in the treatment of subtrochanteric fractures suggested that sliding along the femoral shaft facilitates fracture impaction and stability [4]. Accordingly, in our attempt to increase axial compression along the femoral shaft at the fracture site, we inserted at least two of the distal cortical screws eccentrically in the plate holes [3]. However, excessive impaction at the fracture site, particularly in potentially osteoporotic patients, may be the possible explanation for the reported $1.5 \mathrm{~cm}$ shortening of the injured limb in the aged (75 years old) patient (Fig. 3a-d). Bone grafts in such cases rather than neutralization of the cortical screws may reduce excessive collapse and preserve the femoral length. Wile et al. [13] reported two mal-unions in their series, and Habernek et al. [14] in their study reported two patients with a combination of varus and leg shortening.

Of 37 cases of the current study, no implant failure was reported. Comparison of implant failure with other studies shows that Wile et al. [13] reported no implant failures in 25 subtrochanteric fractures treated with high angle compression hip screws. However, Lunsjö et al. [4] reported three implant failures in 32 fractures treated with the DHS, and Parker et al. [15] reported six failures of fixation in the 74 fractures treated with the sliding hip screw. Habernek et al. [14] reported three implant failures in 14 subtrochanteric fractures treated with the DHS. The authors attributed the implant failures to type of the fracture (e.g. type V) [4], inaccurate placement, or attempting weight bearing too early [14].
In the present study, all fractures were united in a mean time of 11.64 weeks. However, the mean time to union in the Wile et al. [13] series was 3.6 months, and Habernek et al. [14] reported an average 3 months to bone healing in their series. We consider that the absence of implant failure and relatively faster bone healing in the present study can be attributed to the protocol for fracture fixation; i.e., predetermined fracture stabilization with preservation of vascularity of the comminuted fragment and retention of unblocked dynamization during the DHS application.

The strength of the present study is twofold: (1) most of the fractures were of types IIIB and IV (89.1\%). The common denominator in these fracture types is medial cortical comminution, which can result in a lack of stability after internal fixation. (2) This study presented the biomechanics of the DHS in a simplified manner. However, there are two weaknesses: (1) relatively small number of the females (13.5\%); (2) absence of type I and type II C subtrochanteric fractures.

Given the rarity of this injury, it would require a large multicenter, randomized, controlled trial to power a study adequately to compare the relative merits of our approach with other techniques.

\section{Conclusion}

The DHS allows good fixation of a fracture that extends into the piriformis fossa, e.g., type $\mathrm{V}$ subtrochantericintertrochanteric fracture and the long subtrochanteric fractures that extend to supracondylar area of the femur.

Although eccentric placement of at least two cortical screws in the plate holes enhanced axial compression along the femoral shaft, in the potentially osteoporotic patient it may have produced excessive impaction and shortening of the injured limb.

An orthopaedic table and skeletal traction are not necessary for the DHS application; draping the limb free facilitated conversion of the comminuted fracture into a two-part fracture and restoration of the medial buttress with preservation of soft tissue attachment to the bone fragments.

In our opinion, technical optimization of the DHS application nullifies the requirement for evolvement of its design.

\section{References}

1. Kyle RF (1994) Fractures of the proximal part of the femur. J Bone Joint Surg Am 76:924-950

2. Seinsheimer F (1978) Subtrochanteric fractures of the femur. J Bone Joint Surg Am 60A:300-306 
3. Lavelle David G (2003) Campbell's operative orthopaedics, 10th edn. Mosby, St. Louis, p 2897

4. Lunsjö K, Ceder L, Tidermark J et al (1999) Extramedullary fixation of 107 subtrochanteric fractures: A randomized multicenter trial of the Medoff sliding plate versus 3 other screw-plate systems. Acta Orthop Scand 70(5):459-466

5. Parker MJ (1996) Trochanteric hip fractures. Fixation failure commoner with femoral medialization, a comparison of 101 cases. Acta Orthop Scand 67(4):329-332

6. Madsen JE, Naess L, Aune AK, Ahlo A, Ekeland A, Strømsøe K (1998) Dynamic hip screw with trochanteric stabilizing plate in the treatment of unstable proximal femoral fractures: a comparative study with the gamma nail and compression hip screw. J Orthop Trauma 12(4):241-248

7. Miedel R, Ponzer S, Törnkvist $\mathrm{H}$ et al (2005) The standard Gamma nail or the Medoff sliding plate for unstable trochanteric and subtrochanteric fractures. A randomized, controlled trial. J Bone Joint Surg [Br] 87-B:68-75

8. Katz S, Ford A, Moskowitz R, Jackson B, Jaffe M (1963) Studies of illness in the aged: the index of ADL: a standardized measure of biological and psychological function. JAMA 185:94-99
9. Pajarinen J, Lindahl J et al (2005) Pertrochanteric femoral fractures treated with a dynamic hip screw or a proximal femoral nail. J Bone Joint Surg [Br] 87B:76-81

10. DeLee JC (1996) Subtrochanteric fracture. In: Rockwood and Green's fracture in adults, chap 26, 4th edn. Lippincott-Raven, USA

11. Waddell JP (1979) Subtrochanteric fractures of the femur: a review of 130 patients. J Trauma 19:582-592

12. Davis PH, Frymoyer JW (1969) The lateral position in the surgical management of intertrochanteric and subtrochanteric fractures of the femur. J Bone Joint Surg Am 51:1128-1134

13. Wile PB, Panjabi MM, Southwick WO (1983) Treatment of subtrochanteric fractures with a high-angle compression hip screw. Clin Orthop 175:72

14. Habernek H, Schmid L, Frauenschuh E (2000) Sport related proximal femoral fractures: a retrospective review of 31 cases treated in an eight year period. Br J Sports Med 34:54-58

15. Parker MJ, Dutta BK, Sivaji C, Pryor GA (1997) Subtrochanteric fractures of the femur. Injury 28(2):91-95 